\title{
Intravitreal dobesilate injection for macular oedema secondary to branch retinal vein occlusion
}

\begin{abstract}
We describe a patient with macular oedema secondary to occlusion of two branches of the central retinal vein, who has been treated with a single intravitreal injection of dobesilate. Three months after treatment, visual acuity reached 1 , with an anatomical resolution of the macular oedema and the retinal haemorrhage. To our knowledge this is the first case reporting a resolution of macular oedema associated to branch retinal vein occlusions after a single intravitreal injection of dobesilate.
\end{abstract}

Keywords: macular oedema, branch retinal vein occlusion, dobesilate, fibroblast growth factor

\author{
Volume 4 Issue I - 2016 \\ Cuevas Pedro, ${ }^{1,2}$ Outeiriño Luis Antonio, ${ }^{2}$ \\ Azanza Carlos, ${ }^{2}$ Angulo Javier, ${ }^{3}$ Giménez- \\ Gallego Guillermo ${ }^{4}$ \\ 'Universidad Alfonso X, Spain \\ 2Departamento de Oftalmología, Hospital de Día Pío XII, Spain \\ ${ }^{3}$ Departamento de Investigación, Hospital Universitario Ramón \\ y Cajal, Spain \\ ${ }^{4}$ Departamento de Estructura y Función de Proteínas, Centro \\ de Investigaciones Biológicas, Spain
}

Correspondence: NCuevas Pedro, Facultad de Medicina, Universidad Alfonso X, Madrid, Spain,

Email pedro.cuevas44@gmail.com

Received: November 04, 2015 | Published: February 03, 2016

\section{Introduction}

We describe a patient with macular oedema secondary to occlusion of two branches of the central retinal vein, who has been treated with a single intravitreal injection of dobesilate. Three months after treatment, visual acuity reached 1 , with an anatomical resolution of the macular oedema and the retinal haemorrhage. To our knowledge this is the first case reporting a resolution of macular oedema associated to branch retinal vein occlusions after a single intravitreal injection of dobesilate.

\section{Case presentation}

A 68-years old woman presented with complaining of blurry spots and floaters in her left eye for three days. The day of presentation to our service, her best-corrected visual acuity (BCVA) was 0.25 OS. Intraocular pressure was $12 \mathrm{~mm} \mathrm{Hg}$ OS by tonometry. Left anterior segment was normal. Funduscopy exam shows key signs of branch retinal veins occlusion (BCRVO): macular oedema and retinal haemorrhages in the sector dependent of the inferior temporal vein and the superior nasal vein. These veins depicted dilated and tortuous contour (Figure 1). Spectral domain optical coherence tomography (SD-OCT) examination showed cystoid macular oedema and outer layer retinal atrophy.

Treatment with dobesilate was recommended. After approval of our Institutional Ethical Committee, the patient signed an informed consent form, which includes a comprehensive description of the proposed procedure. Using a standard protocol, the patient received an intravitreal solution of dobesilate $(150 \mu l)$ in her left eye under sterile conditions, following the International Guidelines for intravitreal injections. ${ }^{1}$ Dobesilate was administered as a $12.5 \%$ solution of diethylammonium 2,5-dihydroxybenzenesulfonate (etamsylate. Dicynone $^{\circledR}$ Sanofi-Aventis. Paris France). Over 3months follow-up, the haemorrhage signs gradually disappeared (Figure 1), and the SD-OCT scans showed a progressive normalization of the retinal architecture with the disappearance of macular oedema (Figure 1). At the end of this period of three months, the patient's BCVA had reached a value of 1 . No ocular side effects were observed upon the administration of dobesilate or during the following days.

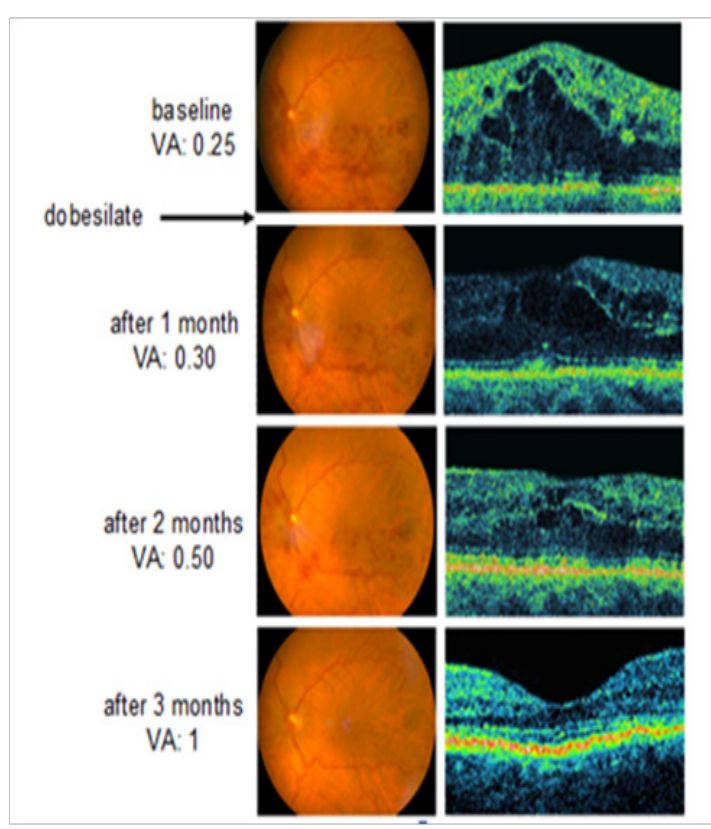

Figure I Sequence of fundus photographs (left row) and spectral-domain optical coherence tomography scans (right row) of a patient with nasal superior and temporal inferior retinal veins occlusion treated with a single intravitreal dobesilate injection. Note the resolution of macular oedema and retinal haemorrhage, as well as improvement of visual acuity (VA) expressed in decimal units.

\section{Discussion}

Retinal vein occlusion (RVO) that can occur either on the central 
retinal vein (CRVO) or branch of the retinal veins (BRVO) is caused by thrombus formation, external compression or disease of the vein wall. Population based studies suggest that about 16 million adults are affected by RVO. ${ }^{2}$ Macular oedema caused by leakage of fluid from capillaries in the central macular area and macular ischemia subsequent to RVO result in significant visual loss. Neovascularization caused by retinal ischemia also leads to visual impairment as a late complication of RVO, and results in vitreous haemorrhage and neovascular glaucoma. Poor visual outcome is due to damage to macular photoreceptors from the chronic oedema, poor perfusion of perifoveolar capillaries, or both. It is generally known that macular oedema and intraretinal haemorrhage occurring in BRVO usually disappear within 6 to 12 months, but clearing of haemorrhages is not accompanied with normal vision; indeed visual acuity improves in $43 \%$, remain static in $39 \%$ and worsen in $18 \%$ of patients. ${ }^{3}$ The main purpose of the BRVO treatment using anti-angiogenic and anti-inflammatory drugs is to decrease the duration of oedema and, thus, prevent photoreceptors damage, whether or not spontaneous improvement occurs.

Clinical evidence supports a role of chronic inflammation in the development of RVO. ${ }^{4,5}$ Ischemia, endothelial damage, cellular hypoxia, with subsequent inflammation, run parallel to elevated levels of fibroblast growth factor (FGF), a first row pro-inflammatory peptide. ${ }^{6-10}$ Previously, we have reported the efficacy of a single intravitreal injection of dobesilate, a FGF inhibitor, ${ }^{11}$ in several inflammatory- and angiogenesis-dependent retinal degenerative diseases. ${ }^{12,13}$ Furthermore, we have reported that neovascular growth induced by FGF and the accompanying bleeding can be suppressed by inhibitors of FGF. ${ }^{14-16}$ In this case report we add new evidence of the antioedematous effect of dobesilate in a patient with CRVO. Consequently, it seems that inhibition of FGF could be an appropriate treatment to prevent retinal injuries in the aftermath of haemorrhages.

\section{Acknowledgements}

None.

\section{Conflict of interest}

The author declares no conflict of interest.

\section{References}

1. Aiello LP, Brucker AJ, Chang S, et al. Evolving guidelines for intravitreous injections. Retina. 2004;24:S3-S19.

2. Rogers SL, McIntosh RL, Lim L, et al. Natural history of branch retina vein occlusion:an evidence-based systematic review. Ophthalmology. 2010;117:1094-1101.
3. Wetzig PC. The treatment of acute branch vein occlusion by photocoagulation. Am J Ophthalmol. 1979;87(1):65-73.

4. Park KS, Kim JW, An JH, et al. Elevated plasma pentraxin 3 and its association with retinal vein occlusion. Korean $J$ Ophthalmol. 2014;28(6):460-465.

5. Noma H, Mimura T, Masahara H, et al. Pentraxin 3 and other inflammatory factors in central retinal vein occlusion and macular edema. Retina. 2014;34(2):352-359.

6. Presta M, Andrés G, Leali D, et al. Inflammatory cells and chemokines sustain FGF2-induced angiogenesis. Eur Cytokine Netw. 2009;20:3950 .

7. Stewart MW. The expanding role of vascular endothelial growth factor inhibitors in ophthalmology. Mayo Clin Proc. 2012;87(1):77-88.

8. Seo JH, Yu JH, Suh H, et al. Fibroblast growth factor-2 induced by enriched environment enhances angiogenesis and motor function in chronic hypoxic-ischemic brain injury. PLoS One. 2013;8(9):e74405.

9. Yafai Y, Iandiev I, Lange J, et al. Basic fibroblast growth factor contributes to a shift in the angioregulatory activity of retinal glial (Müller) cells. PLoS One. 2013;8(7):e68773.

10. Wiktorowska-Owczarek A, Namiecinska M, Owczarek J. The effect of ibuprofen on bFGF, VEGF secretion and cell proliferation in the presence of LPS in HMEC-1 cells. Acta Pol Pharm. 2015;72(5):889-894.

11. Fernández IS, Cuevas P, Angulo, López-Navajas P, et al. Gentisic acid, a compound associated with plant defense and a metabolite of aspirin, heads a new class of in vivo fibroblast growth factor inhibitors. $J$ Biol Chem. 2010;285(15):11714-11729.

12. Cuevas P, Outeiriño LA, Angulo J, et al. Treatment of dry age-related macular degeneration with dobesilate. BMJ Case Rep. 2012.

13. Cuevas P, Outeiriño LA, Azanza C, et al. Short-term efficacy of intravitreal dobesilate in central serous chorioretinopathy. Eur J Med Res. 2012;17:22.

14. Cuevas P, Carceller F, Reimers D, et al. Inhibition of intra-tumoral angiogenesis and glioma growth by the fibroblast growth factor inhibitor 1,3,6-naphthalenetrisulfonate. Neurol Res. 1999;21:481-487.

15. Cuevas P, Outeiriño LA, Azanza C, Angulo J, et al. Case report: resolution of submacular haemorrhage secondary to exudative agerelated macular degeneration after a single intravitreal dobesilate injection. F1000 Res. 2013;2:271

16. Cuevas P, Outeiriño LA, Azanza C, et al. Dramatic resolution of vireous hemorrhage alter an intravitreal injection of dobesilate. Mil Med Res. $2015 ; 2: 23$ 